\title{
Potencial de Espécies Vegetais PaRa a Remediação do HeRbicida TRIFLOXYSULFURON-SODIUM ${ }^{1}$
}

\author{
Potential of Plant Species for Remediation of Trifloxysulfuron-Sodium \\ PROCÓPIO, S.O. ${ }^{2}$, SANTOS, J.B. ${ }^{3}$, SILVA, A.A. ${ }^{4}$, PIRES, F.R. ${ }^{2}$, RIBEIRO JÚNIOR, J.I. ${ }^{5}$ e \\ SANTOS, E.A. ${ }^{6}$
}

\begin{abstract}
RESUMO - Este trabalho teve como objetivo avaliar a eficiência de espécies vegetais na remediação do herbicida trifloxysulfuron-sodium em solos, utilizando o feijão (Phaseolus vulgaris) como planta indicadora. Os tratamentos foram compostos pela combinação entre as espécies Calopogonium mucunoides, Crotalaria juncea, Crotalaria spectabilis, Vicia sativa, Cajanus cajan, Canavalia ensiformis, Medicago sativa, Dolichus lab lab, Penisetum glaucum, Stylosantes guianensis, Mucuna deeringiana, Mucuna cinereum, Mucuna aterrima, Raphanus sativus e Lupinus albus. Todas as espécies foram semeadas em vasos no dia seguinte à aplicação do trifloxysulfuron-sodium em três doses $\left(0,00 ; 3,75\right.$; e 15,00 $\left.\mathrm{g} \mathrm{ha}^{-1}\right)$. Após 80 dias da semeadura, as espécies vegetais foram cortadas na altura do coleto e a parte aérea destas descartada. A seguir, foi realizada a semeadura do feijão (cultivar Pérola). Aos 45 dias após a emergência das plantas de feijão avaliaram-se a altura e a massa seca da parte aérea das plantas. Melhor eficiência na descontaminação do trifloxysulfuron-sodium em solo foi obtida pelas espécies M. aterrima e C. ensiformis.
\end{abstract}

Palavras-chave: fitorremediação, descontaminação, adubos verdes, solos.

\begin{abstract}
This work aimed to evaluate the efficiency of vegetable species in the remediation of the herbicide trifloxysulfuron-sodium in soils using the common bean (Phaseolus vulgaris) as a bio indicator. The treatments were composed by the combination of the species Calopogonium mucunoides, Crotalaria juncea, Crotalaria spectabilis, Vicia sativa, Cajanus cajan, Canavalia ensiformis, Medicago sativa, Dolichus lab lab, Penisetum glaucum, Stylosantes guianensis, Mucuna deeringiana, Mucuna cinereum, Mucuna aterrima, Raphanus sativus and Lupinus albus, sown in vases the day following application of the herbicide at three doses (0.00; 3.75; and $15.00 \mathrm{~g} \mathrm{ha}^{-1}$.) Eighty days after sowing, the plants were cut and the shoot discarded, followed by bean ("Perola" cultivar) sowing. At 45 days post emergence, bean height and shoot dry matter were evaluated. Greater efficiency in trifloxysulfuron-sodium decontamination in soil was obtained with the species $\boldsymbol{M}$. aterrima and $\mathbf{C}$. ensiformis.
\end{abstract}

Key words: phytoremediation, decontamination, green manure, soils.

\section{INTRODUÇÃO}

Herbicidas que apresentam longa persistência em solo podem tornar inviável a rotação de culturas, técnica esta essencial quando se visa cultivo sustentável. Entre esses herbicidas encontra-se o trifloxysulfuronsodium, recentemente registrado no País, o qual vem sendo amplamente utilizado na cultura do algodão, aplicado em pós-emergência inicial, e também na cultura da cana em mistura com o ametryn. Mesmo utilizado em

Recebido para publicação em 13.9.2004 e na forma revisada em 21.4.2005.

2 Professor do Departamento de Agronomia, FESURV, Rio Verde-GO. ${ }^{3}$ Estudante de doutorado do Departamento de Fitotecnia da Universidade Federal de Viçosa - UFV, <jbarbosasantos@yahoo.com.br>; ${ }^{4}$ Professor do Departamento de Fitotecnia da UFV; ${ }^{5}$ Professor do Departamento de Informática da UFV. ${ }^{6}$ Acadêmico do curso de Agronomia da UFV. 
baixas concentrações (em torno de $7,5 \mathrm{~g} \mathrm{ha}^{-1}$ ), o período de espera para o plantio de culturas sensiveis pode chegar a oito meses, a contar de sua aplicação. Esse fato pode limitar a utilização do produto em áreas onde o agricultor cultiva, por exemplo, feijão no inverno.

$\mathrm{Na}$ busca de alternativas para minimizar esse problema, tem-se difundido o uso de plantas tolerantes para a remoção de determinados produtos. Essa técnica é conhecida como fitorremediação, e seu emprego tem sido indicado em áreas contaminadas com substâncias orgânicas e inorgânicas (Cunningham et al., 1996).

A fitorremediação baseia-se na tolerância que algumas espécies exibem a determinados compostos ou mecanismos de ação. No caso de compostos orgânicos, a tolerância pode ser resultante de processos como a translocação diferencial (maior ou menor) para outros tecidos da planta, com subseqüente volatilização; degradação parcial ou completa; e transformação em compostos menos tóxicos, combinados e/ou ligados a tecidos das plantas (compartimentalização) (Accioly \& Siqueira, 2000; Scramin et al., 2001; Pires et al., 2003). Em geral, a maioria dos compostos orgânicos passa por transformações nas células das plantas antes de serem isolados em vacúolos ou ligarem-se a estruturas celulares insolúveis, como a lignina (Macek et al., 2000).

A fitorremediação também pode ocorrer pela liberação de exsudatos radiculares, que estimulam a atividade microbiana (fitoestimulação), degradando o composto no solo e caracterizando, em algumas espécies vegetais, a aptidão rizosférica para a biorremediação de compostos tóxicos (Cunningham et al., 1996; Pires et al., 2003).

Apesar da limitação que existe para fitorremediar herbicidas, por serem estes, muitas vezes, tóxicos às plantas, recentes pesquisas têm relatado o uso de plantas na biodegradação de pesticidas. Os herbicidas para os quais têm sido observados resultados positivos quanto à fitorremediação são principalmente atrazine e metolachlor (Anderson et al., 1994; Anderson \& Coats, 1995; Perkovich et al., 1996; Rice et al., 1997; Pires et al., 2003), e a espécie mais promissora tem sido Kochia scoparia. Estudos envolvendo solo rizosférico dessa espécie evidenciaram que a degradação de metolachlor e, sobretudo, de atrazine foi maior, comparada à de solos não vegetados por K. scoparia. Estudos com trifluralin (Anderson et al., 1994), simazine (Wilson et al., 1999, 2000) e 2,4,5-T (Boyle \& Schann, 1998) também têm sido relatados.

No Brasil, poucos trabalhos são relatados. Scramin et al. (2001) identificaram plantas daninhas persistentes, supostamente tolerantes aos herbicidas mais comumente utilizados nas áreas de cana-de-açúcar do Estado de São Paulo.

Para Pires et al. (2003), a fitorremediação de herbicidas requer ação conjunta de profissionais de diversas áreas para que se obtenha sucesso na identificação de espécies capazes de atuar na descontaminação de solos, principalmente nas condições edafoclimáticas brasileiras.

Assim, para utilização da fitorremediação como alternativa para descontaminação de solos e, no caso do trifloxysulfuron-sodium, para minimizar sua toxicidade em espécies sensiveis cultivadas em rotação, torna-se necessária a seleção de plantas que sejam capazes de degradá-lo/imobilizá-lo no solo ou na planta, principalmente se esta característica estiver também associada a outros beneficios ao solo, como é o caso dos adubos verdes.

Este trabalho teve como objetivo avaliar o potencial de espécies vegetais na remediação do herbicida trifloxysulfuron-sodium em solos, utilizando o feijão como planta indicadora.

\section{MATERIAL E MÉTODOS}

O experimento foi realizado utilizando como substrato, solo classificado como Argissolo Vermelho-Amarelo, de textura argilo-arenosa, cuja caracterização físico-química encontrase na Tabela 1.

Os tratamentos foram compostos pela combinação entre as espécies vegetais Calopogonium mucunoides (calopogônio), Crotalaria juncea (crotalária), Crotalaria spectabilis (crotalária), Vicia sativa (ervilhaca), Cajanus cajan (feijão-guandu), Canavalia ensiformis (feijão-de-porco), Medicago sativa (alfafa), Dolichus lab lab (lablabe), Penisetum glaucum (milheto), Stylosantes guianensis (mineirão), Mucuna deeringiana (mucuna-anã), 
Tabela 1 - Composição físico-química da camada arável $(0-20 \mathrm{~cm})$ do solo Argissolo Vermelho-Amarelo utilizado no experimento

\begin{tabular}{|c|c|c|c|c|c|c|c|c|c|c|}
\hline \multicolumn{11}{|c|}{ Análise granulométrica $\left(\mathrm{dag}_{\mathrm{kg}}{ }^{-1}\right)$} \\
\hline \multicolumn{2}{|c|}{ Argila } & \multicolumn{2}{|r|}{ Silte } & \multicolumn{2}{|c|}{ Areia fina } & \multicolumn{2}{|c|}{ Areia grossa } & \multicolumn{3}{|c|}{ Classificação textural } \\
\hline \multicolumn{2}{|c|}{39} & \multicolumn{2}{|r|}{11} & \multicolumn{2}{|r|}{17} & \multicolumn{2}{|c|}{33} & \multicolumn{3}{|c|}{ Argilo-arenosa } \\
\hline \multicolumn{11}{|c|}{ Análise Química } \\
\hline $\mathrm{pH}$ & $\mathrm{P}$ & $\mathrm{K}^{+}$ & $\mathrm{H}+\mathrm{Al}$ & $\mathrm{Al}^{3+}$ & $\mathrm{Ca}^{2+}$ & $\mathrm{Mg}^{2+}$ & $\mathrm{CTC}_{\text {total }}$ & $\mathrm{V}$ & M & MO \\
\hline$\left(\mathrm{H}_{2} \mathrm{O}\right)$ & \multicolumn{2}{|c|}{$\left.(\mathrm{mg} \mathrm{dm})^{-3}\right)$} & \multicolumn{5}{|c|}{$\left(\mathrm{cmol}_{\mathrm{c}} \mathrm{dm}^{-3}\right)$} & \multicolumn{2}{|c|}{$(\%)$} & $\left(\right.$ dag $\left.\mathrm{kg}^{-1}\right)$ \\
\hline 5,8 & 1,4 & 123 & 4,3 & 0,0 & 3,8 & 1,4 & 9,81 & 56 & 0 & 2,18 \\
\hline
\end{tabular}

* Análises realizadas nos Laboratórios de Análises Físicas e Químicas de Solo do Departamento de Solos da UFV, segundo a metodologia descrita pela Empresa Brasileira de Pesquisa Agropecuária - Embrapa (1997).

Mucuna cinereum (mucuna-cinza), Mucuna aterrima (mucuna-preta), Raphanus sativus (nabo-forrageiro) e Lupinus albus (tremoçobranco), semeadas anteriormente à cultura do feijão (Phaseolus vulgaris), mais um tratamento controle - sem cultivo prévio, e de três doses do herbicida trifloxysulfuron-sodium $\left(0,00 ; 3,75\right.$; e $\left.15,00 \mathrm{~g} \mathrm{ha}^{-1}\right)$. A escolha das espécies e das doses do trifloxysulfuron-sodium baseou-se em ensaios preliminares de tolerância a este herbicida.

O delineamento experimental utilizado foi o de blocos casualizados em esquema fatorial $16 \times 3$, com três repetições. A unidade experimental foi constituída de um vaso de polietileno, contendo $3 \mathrm{dm}^{3}$ de solo adubado com $600 \mathrm{~kg} \mathrm{ha}^{-1}$ da fórmula 4-14-8 antes do preenchimento deles. Após essa etapa, os vasos foram umedecidos e, após 24 horas, procedeuse à aplicação em pré-emergência do herbicida. Para isso, utilizou-se um pulverizador costal de precisão, aplicando-se o equivalente a $260 \mathrm{~L} \mathrm{ha}^{-1}$ de calda.

A semeadura das espécies vegetais foi feita no dia seguinte à aplicação do trifloxysulfuron-sodium. Após a emergência das plantas foi realizado desbaste, deixando quatro vezes a densidade recomendada de cada espécie, com base no uso das espécies na adubação verde. Os vasos foram mantidos sob irrigação, a fim de se conservar a umidade do solo em torno de $80 \%$ da capacidade de campo.

Transcorridos 80 dias da semeadura, as espécies vegetais foram cortadas na altura do coleto e a parte aérea destas descartada. A seguir, foi realizada a semeadura do feijão (cultivar Pérola). Após a emergência das plantas de feijão, efetuou-se desbaste, deixandose duas plantas por vaso.

Aos 45 dias após a emergência das plantas de feijão, avaliou-se a altura de plantas, adotando-se como base para medição o meristema apical das plantas e a massa seca da parte aérea destas, obtida por meio de pesagem do material colhido, secado em estufa de circulação forçada $\left(70 \pm 2{ }^{\circ} \mathrm{C}\right)$ por 72 horas.

Todas as variáveis que atenderam às pressuposições de normalidade e homogeneidade das variâncias, por meio dos testes de Lilliefors e de Cochran, respectivamente, foram submetidas à análise de variância. O efeito entre as doses do trifloxysulfuron-sodium dentro de cada espécie vegetal foi avaliado por análise de regressão, a 5 ou 1\% de significância, e o efeito entre as espécies dentro de cada dose do herbicida, por meio do critério de Scott-Knott a $5 \%$ de significância.

\section{RESULTADOS E DISCUSSÃO}

\section{Altura de Plantas}

Constatou-se efeito significativo $(\mathrm{P}<0,01$ ou $\mathrm{P}<0,05$ ) das doses de trifloxysulfuron-sodium sobre a altura das plantas de feijão quando semeadas em seqüência às espécies $C$. juncea, C. spectabilis, C. cajan, P. glaucum, S. guianensis, $M$. deeringiana, $L$. albus e, também, quando não houve cultivo anterior (controle) (Figura $1 \mathrm{e}$ Tabela 2). Para esses tratamentos, o aumento das doses resultou em plantas de menor altura. A intensidade desse efeito variou com a espécie testada (Figura 1). Não foi detectado 

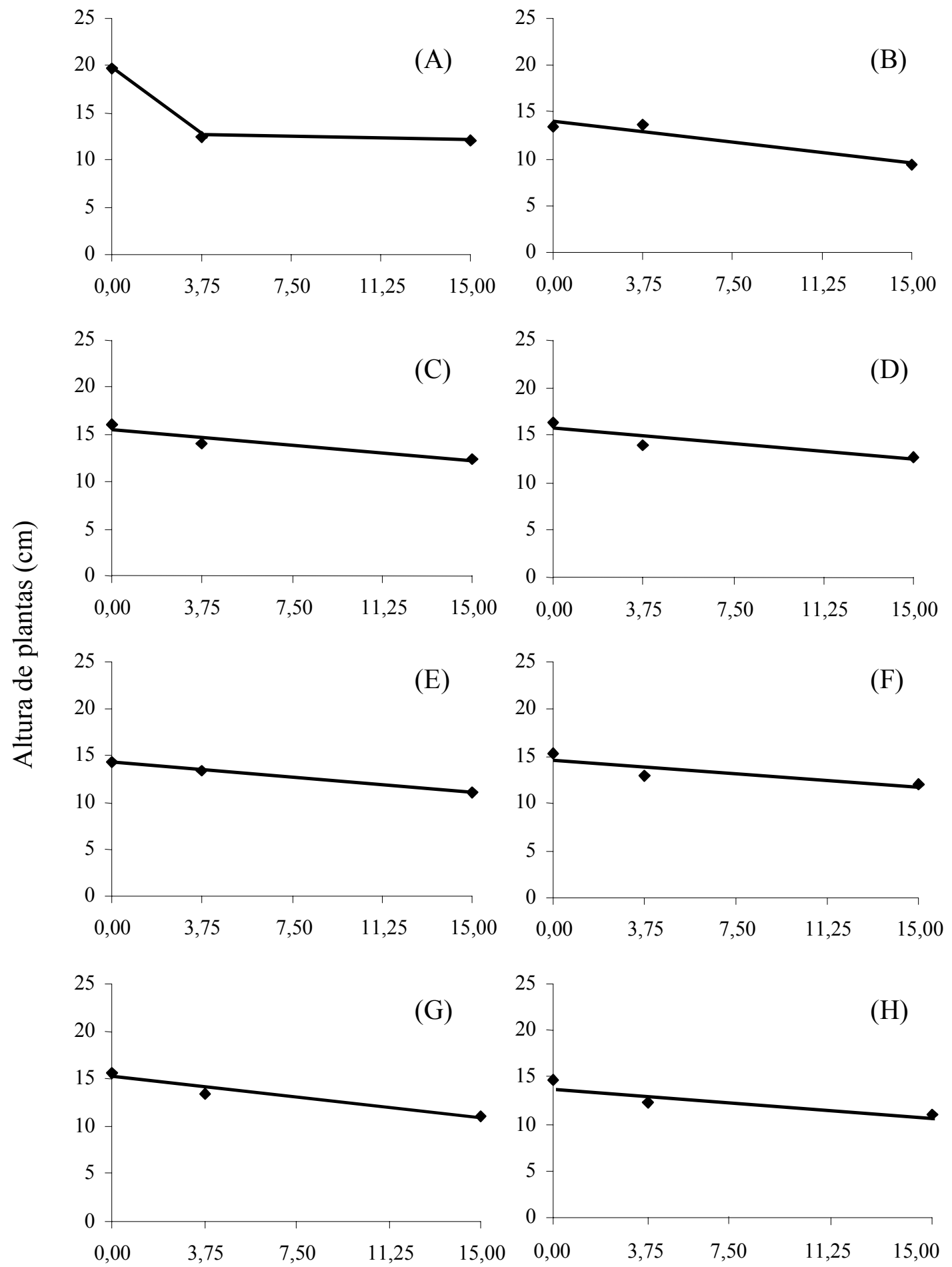

Dose de trifloxysulfuron-sodium $\left(\mathrm{g} \mathrm{ha}^{-1}\right)$

Figura 1 - Estimativa das alturas de plantas de feijão aos 45 dias após a emergência, depois da semeadura de diversas espécies vegetais [controle - sem cultivo (A), Crotalaria juncea (B), Crotalaria spectabilis (C), Cajanus cajan (D), Penisetum glaucum (E), Stylosantes guianensis $(\mathrm{F})$, Mucuna deeringiana $(\mathrm{G})$ e Lupinus albus $(\mathrm{H})]$ em solos submetidos a três doses de trifloxysulfuronsodium. 
Tabela 2 - Efeito do plantio anterior de diversas espécies vegetais sobre a altura de plantas de feijão aos 45 dias após a emergência, semeadas em solo submetido a três doses do herbicida trifloxysulfuron-sodium (D). Viçosa-MG, 2003

\begin{tabular}{|c|c|c|c|c|c|}
\hline \multirow{3}{*}{ Espécie fitorremediadora } & \multicolumn{3}{|c|}{ Dose de trifloxysulfuron-sodium $\left(\mathrm{g} \mathrm{ha}^{-1}\right)$} & \multirow{3}{*}{ Equação de regressão } & \multirow{3}{*}{$\mathrm{R}^{2}(\%)$} \\
\hline & 0,00 & 3,75 & 15,00 & & \\
\hline & \multicolumn{3}{|c|}{ Altura de plantas de feijão $(\mathrm{cm})$} & & \\
\hline Sem cultivo prévio & 19,67 a & $12,33 \mathrm{~b}$ & $12,00 \mathrm{~b}$ & $\begin{array}{l}\hat{\mathrm{Y}}=19,67-1,956 * \mathrm{D}(0 \leq \mathrm{D} \leq 3,92) \mathrm{e} \\
\overline{\mathrm{Y}}=12(3,92 \leq \mathrm{D} \leq 15)\end{array}$ & 100,00 \\
\hline Medicago sativa & $14,00 \mathrm{~b}$ & $14,00 \mathrm{~b}$ & $11,67 \mathrm{~b}$ & $\bar{Y}=13,22$ & - \\
\hline Calopogonium mucunoides & $14,00 \mathrm{~b}$ & $12,00 \mathrm{~b}$ & $11,67 \mathrm{~b}$ & $\overline{\mathrm{Y}}=12,56$ & - \\
\hline Crotalaria juncea & $13,33 \mathrm{~b}$ & $13,67 \mathrm{~b}$ & $9,33 \mathrm{~b}$ & $\hat{\mathrm{Y}}=13,9487-0,294017^{* *} \mathrm{D}$ & 90,59 \\
\hline Crotalaria spectabilis & $16,00 \mathrm{~b}$ & $14,00 \mathrm{~b}$ & $12,33 \mathrm{~b}$ & $\hat{\mathrm{Y}}=15,5-0,222222 * \mathrm{D}$ & 89,29 \\
\hline Vicia sativa & $11,67 \mathrm{~b}$ & $11,67 \mathrm{~b}$ & $10,33 \mathrm{~b}$ & $\bar{Y}=11,22$ & - \\
\hline Cajanus cajan & $16,33 \mathrm{~b}$ & $14,00 \mathrm{~b}$ & $12,67 \mathrm{~b}$ & $\hat{\mathrm{Y}}=15,6795-0,215385^{*} \mathrm{D}$ & 82,07 \\
\hline Canavalia ensiformis & $18,33 \mathrm{a}$ & $18,00 \mathrm{a}$ & 17,17 a & $\bar{Y}=17,83$ & - \\
\hline Dolichus lab lab & $14,00 \mathrm{~b}$ & $12,67 \mathrm{~b}$ & $13,83 \mathrm{~b}$ & $\bar{Y}=13,50$ & - \\
\hline Penisetum glaucum & $14,33 \mathrm{~b}$ & $13,33 \mathrm{~b}$ & $11,00 \mathrm{~b}$ & $\hat{\mathrm{Y}}=14,2564-0,218803 * \mathrm{D}$ & 99,71 \\
\hline Stylosantes guianensis & $15,33 \mathrm{~b}$ & $13,00 \mathrm{~b}$ & $12,00 \mathrm{~b}$ & $\hat{\mathrm{Y}}=14,641-0,191453^{*} \mathrm{D}$ & 76,34 \\
\hline Mucuna deeringiana & $15,67 \mathrm{~b}$ & $13,33 \mathrm{~b}$ & $11,00 \mathrm{~b}$ & $\hat{\mathrm{Y}}=15,1282-0,287179^{* *} \mathrm{D}$ & 92,31 \\
\hline Mucuna cinereum & $16,00 \mathrm{~b}$ & $15,67 \mathrm{a}$ & $14,00 \mathrm{~b}$ & $\bar{Y}=15,22$ & - \\
\hline Mucuna aterrima & $19,33 \mathrm{a}$ & $19,00 \mathrm{a}$ & $19,00 \mathrm{a}$ & $\bar{Y}=19,11$ & - \\
\hline Raphanus sativus & $12,67 \mathrm{~b}$ & $11,67 \mathrm{~b}$ & $11,33 \mathrm{~b}$ & $\overline{\mathrm{Y}}=11,89$ & - \\
\hline Lupinus albus & $14,67 \mathrm{~b}$ & $12,33 \mathrm{~b}$ & $11,00 \mathrm{~b}$ & $\hat{\mathrm{Y}}=14,0128-0,215385^{*} \mathrm{D}$ & 82,07 \\
\hline
\end{tabular}

Médias seguidas pela mesma letra, na coluna, não diferem entre si pelo critério de Scott-Knott $(\mathrm{P}>0,05)$.

* Significativo pelo teste $\mathrm{t}(\mathrm{P}<0,05)$; $\mathrm{e}^{* *}$ significativo pelo teste $\mathrm{t}(\mathrm{P}<0,01)$.

efeito das doses do herbicida sobre a altura das plantas quando, anteriormente ao feijão, foram cultivados $M$. sativa, C. mucunoides, $V$. sativa, C. ensiformis, D. lab lab, M. cinereum, $M$. aterrima e $R$. sativus (Tabela 2).

Nos tratamentos que não receberam o trifloxysulfuron-sodium, as únicas espécies semeadas previamente ao feijão que interferiram na altura das plantas desta cultura foram $M$. aterrima e $C$. ensiformis. O cultivo anterior de todas as outras espécies testadas em solo livre do herbicida resultou em diminuição da altura das plantas de feijão, possivelmente devido a efeitos alelopáticos.

Em relação à dose do trifloxysulfuronsodium de 3,75 $\mathrm{g} \mathrm{ha}^{-1}$, aproximadamente metade da dose recomendada pelo fabricante para a cultura do algodão, verificou-se que a maior altura das plantas de feijão foi obtida após seu cultivo em solo que recebeu previamente as espécies $M$. aterrima, C. ensiformis e
M. cinereum, sendo superior ao tratamento controle (Tabela 2). Entretanto, quando o feijão foi cultivado em tratamentos com $15 \mathrm{~g} \mathrm{ha}^{-1} \mathrm{de}$ trifloxysulfuron-sodium, somente $M$. aterrima e C. ensiformis não apresentaram queda na altura desta cultura (Tabela 2). Efeito semelhante foi observado por Anderson et al. (1994), os quais demonstraram que a degradação de atrazine, metolachlor e trifluralin foi maior em solos rizosféricos de Kochia scoparia que em solos não-vegetados.

\section{Biomassa seca da parte aérea (BSPA)}

Quanto ao efeito do trifloxysulfuronsodium sobre o acúmulo de BSPA pelas plantas de feijão, observou-se que ele foi significativo para doses quando a cultura foi semeada em seqüência às espécies C. mucunoides, C. juncea, C. cajan, P. glaucum, S. guianensis, $M$. cinereum, $M$. aterrima, $R$. sativus e $L$. albus e, também, quando não houve cultivo anterior 


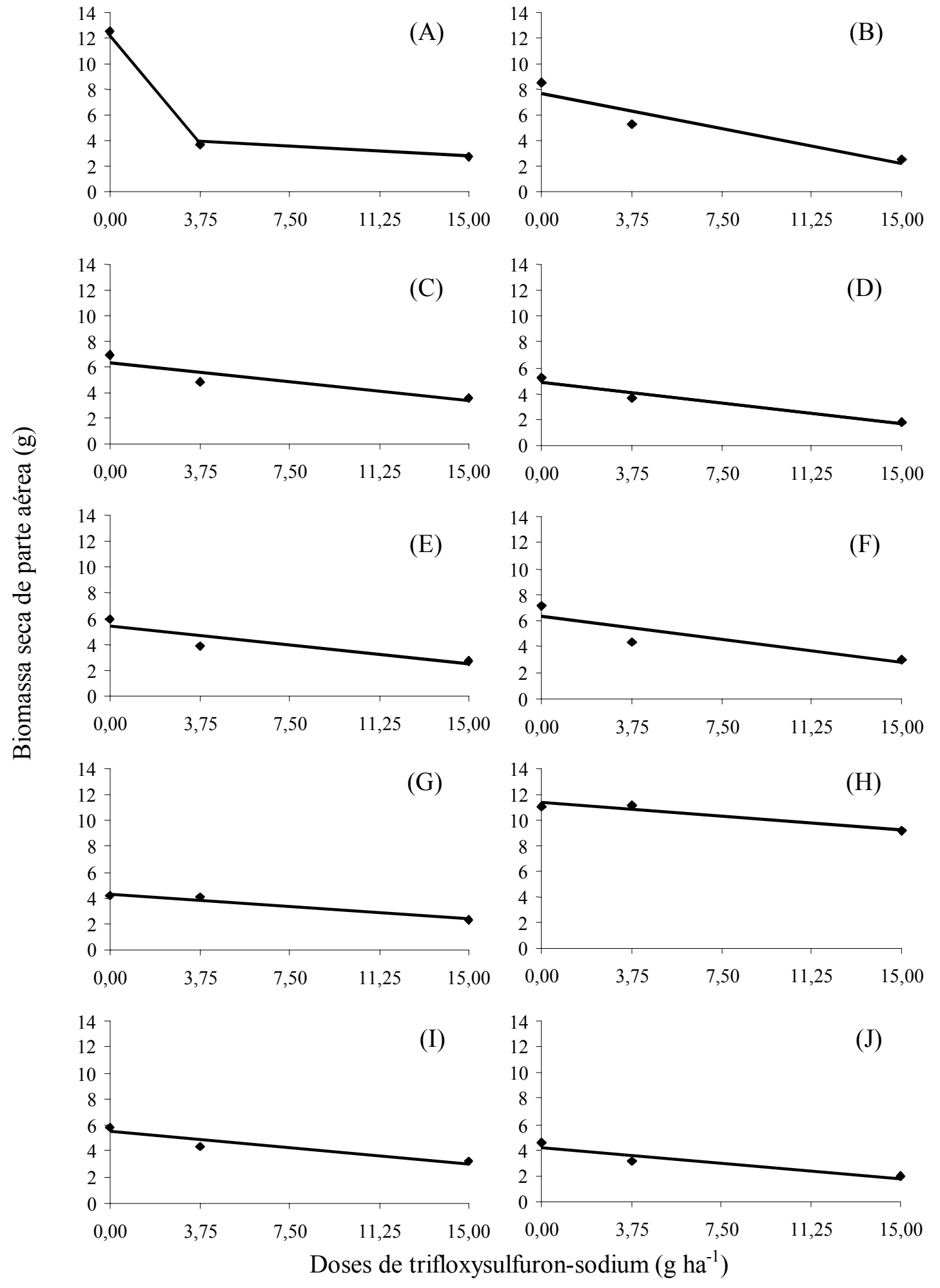

Figura 2 - Estimativas da massa seca de parte aérea de plantas de feijão aos 45 dias após a emergência, depois da semeadura de diversas espécies vegetais [controle - sem cultivo (A), Crotalaria mucunoides (B), Crotalaria juncea (C), Cajanus cajan (D), Penisetum glaucum (E), Stylosantes guianensis (F), Mucuna cinereum (G), Mucuna aterrima (H), Raphanus sativus (I) e Lupinus albus $(\mathrm{J})$ ] em solos submetidos a três doses de trifloxysulfuron-sodium. 
(controle) (Figura 2 e Tabela 3). Nesses tratamentos observou-se diminuição do acúmulo de MSPA com o aumento das doses do herbicida, variando na intensidade da queda de acordo com a espécie testada (Figura 2), com exceção do tratamento controle, que apresentou essa diminuição até, aproximadamente, a dose de 3,75 $\mathrm{g} \mathrm{ha}^{-1}$. Não foi observado efeito das doses do herbicida sobre esta característica avaliada quando se semearam anteriormente à cultura do feijão as espécies $M$. sativa, C. spectabilis, V. sativa, C. ensiformis, D. lab lab e $M$. deeringiana.

Quanto ao efeito das espécies sobre a BSPA de feijão, verificou-se que, à exceção de $M$. aterrima e C. ensiformis, todas interferiram de forma negativa nesta característica (Tabela 3). No entanto, o tratamento com C. mucunoides foi superior àqueles com $M$. sativa, C. juncea, $V$. sativa, D. lab lab, P. glaucum, S. guianensis e $R$. sativus, os quais, ainda assim, diferiram dos tratamentos que tiveram o plantio anterior das espécies C. spectabilis, C. cajan, $M$. deeringiana e $L$. albus. Novamente, a razão mais provável para esses resultados seria a ocorrência de efeitos alelopáticos das plantas previamente semeadas sobre as plantas de feijão, como já relatado anteriormente para a altura de plantas.

No tratamento com a dose de $3,75 \mathrm{~g} \mathrm{ha}^{-1}$ de trifloxysulfuron-sodium, $V$. sativa, apesar de não ter proporcionado BSPA das plantas de feijão igual à de $M$. aterrima e $C$. ensiformes, foi superior aos demais tratamentos. Distinguem-se, ainda, os tratamentos contendo M. sativa, C. mucunoides, C. juncea e D. lab lab dos tratamentos que envolveram $C$. spectabilis, C. cajan, P. glaucum, S. guianensis, $M$. deeringiana, $M$. cinereum, $R$. sativus e L. albus. Esses últimos apresentaram os piores efeitos sobre a BSPA das plantas de feijão nessa dose do herbicida.

Tabela 3 - Efeito do plantio anterior de diversas espécies vegetais sobre a Biomassa seca da parte aérea (BSPA) de plantas de feijão aos 45 dias após a emergência, semeadas em solo submetido a três doses do herbicida trifloxysulfuron-sodium (D). Viçosa-MG, 2003

\begin{tabular}{|c|c|c|c|c|c|}
\hline \multirow{3}{*}{ Espécie fitorremediadora } & \multicolumn{3}{|c|}{ Dose de trifloxysulfuron-sodium $\left(\mathrm{g} \mathrm{ha}^{-1}\right)$} & \multirow{3}{*}{ Equação de regressão } & \multirow{3}{*}{$\mathrm{R}^{2}(\%)$} \\
\hline & 0,00 & 3,75 & 15,00 & & \\
\hline & \multicolumn{3}{|c|}{ BSPA $(\mathrm{cm})$} & & \\
\hline Sem cultivo prévio & $12,51 \mathrm{a}$ & $3,71 \mathrm{~d}$ & $2,72 \mathrm{c}$ & $\begin{array}{l}\hat{\mathrm{Y}}=12,513-2,347^{* *} \mathrm{D}(0 \leq \mathrm{D} \leq 4,17) \mathrm{e} \\
\overline{\mathrm{Y}}=2,72(4,17 \leq \mathrm{D} \leq 15)\end{array}$ & 100,00 \\
\hline Medicago sativa & $6,15 \mathrm{c}$ & $5,77 \mathrm{c}$ & $5,39 \mathrm{~b}$ & $\bar{Y}=5,77$ & - \\
\hline Calopogonium mucunoides & $8,54 \mathrm{~b}$ & $5,29 \mathrm{c}$ & $2,52 \mathrm{c}$ & $\hat{\mathrm{Y}}=7,73423-0,365299^{* *} \mathrm{D}$ & 89,66 \\
\hline Crotalaria juncea & $6,95 \mathrm{c}$ & $4,83 \mathrm{c}$ & $3,53 \mathrm{c}$ & $\hat{\mathrm{Y}}=6,36577-0,201812^{* *} \mathrm{D}$ & 83,43 \\
\hline Crotalaria spectabilis & $4,93 \mathrm{~d}$ & $4,60 \mathrm{~d}$ & $3,47 \mathrm{c}$ & $\overline{\mathrm{Y}}=4,33$ & - \\
\hline Vicia sativa & $7,93 \mathrm{c}$ & $7,29 \mathrm{~b}$ & $6,48 \mathrm{~b}$ & $\bar{Y}=7,23$ & - \\
\hline Cajanus cajan & $5,21 \mathrm{~d}$ & $3,65 \mathrm{~d}$ & $1,74 \mathrm{c}$ & $\hat{\mathrm{Y}}=4,89-0,216889^{* *} \mathrm{D}$ & 95,09 \\
\hline Canavalia ensiformis & $10,82 \mathrm{a}$ & $10,15 \mathrm{a}$ & 9,45 a & $\overline{\mathrm{Y}}=10,14$ & - \\
\hline Dolichus lab lab & $6,76 \mathrm{c}$ & $6,02 \mathrm{c}$ & $5,66 \mathrm{~b}$ & $\bar{Y}=6,15$ & - \\
\hline Penisetum glaucum & $5,99 \mathrm{c}$ & $3,86 \mathrm{~d}$ & $2,70 \mathrm{c}$ & $\hat{\mathrm{Y}}=5,38538-0,192684^{* *} \mathrm{D}$ & 81,08 \\
\hline Stylosantes guianensis & $6,74 \mathrm{c}$ & $4,51 \mathrm{~d}$ & $3,77 \mathrm{c}$ & $\hat{\mathrm{Y}}=6,05154-0,167179^{* *} \mathrm{D}$ & 71,33 \\
\hline Mucuna deeringiana & $3,19 \mathrm{~d}$ & $3,40 \mathrm{~d}$ & $2,66 \mathrm{c}$ & $\bar{Y}=3,08$ & - \\
\hline Мисипа cinereum & $4,13 \mathrm{~d}$ & $4,04 \mathrm{~d}$ & $2,30 \mathrm{c}$ & $\hat{\mathrm{Y}}=4,30372-0,130017 * \mathrm{D}$ & 96,09 \\
\hline Mucuna aterrima & $11,08 \mathrm{a}$ & $11,20 \mathrm{a}$ & 9,18 a & $\hat{\mathrm{Y}}=11,3558-0,138701^{* *} \mathrm{D}$ & 91,43 \\
\hline Raphanus sativus & $5,84 \mathrm{c}$ & $4,35 \mathrm{~d}$ & $3,17 \mathrm{c}$ & $\hat{\mathrm{Y}}=5,45705-0,161128^{* *} \mathrm{D}$ & 88,37 \\
\hline Lupinus albus & $4,56 \mathrm{~d}$ & $3,11 \mathrm{~d}$ & $1,95 \mathrm{c}$ & $\hat{\mathrm{Y}}=4,19231-0,15788^{* *} \mathrm{D}$ & 88,61 \\
\hline
\end{tabular}

Médias seguidas pela mesma letra, na coluna, não diferem entre si pelo critério de Scott-Knott $(\mathrm{P}>0,05)$.

* Significativo pelo teste $\mathrm{t}(\mathrm{P}<0,05)$; $\mathrm{e}^{* *}$ significativo pelo teste $\mathrm{t}(\mathrm{P}<0,01)$. 
Contudo, quando o feijão foi cultivado com C. ensiformis em substrato tratado com até $15 \mathrm{~g} \mathrm{ha}^{-1}$ de trifloxysulfuron-sodium, não se observou redução significativa no acúmulo de BSPA, diferenciando-se das demais espécies, inclusive do tratamento sem cultivo prévio. Este tratamento não diferiu daquele cultivado com $M$. aterrima (Tabela 3). A BSPA das plantas de feijão que receberam o cultivo prévio das plantas de $C$. ensiforme $(9,45 \mathrm{~g})$ e $M$. aterrima $(9,18 \mathrm{~g})$ foi, aproximadamente, três vezes superior à daquele produzido no tratamento controle sem cultivo prévio $(2,72 \mathrm{~g})$, ou seja, sem fitorremediação. Wilson et al. (2000) verificaram que a atividade de simazine em solução foi reduzida a 45 e $34 \%$ em sete dias com a presença de Acorus gramenius e Pontederia cordata, respectivamente, sugerindo serem eficientes na fitorremediação deste herbicida.

Com base nos resultados deste trabalho, conclui-se que as espécies $M$. aterrima e C. ensiformis foram eficientes na descontaminação do herbicida trifloxysulfuron-sodium. O uso dessa tecnologia poderá resultar em maior segurança do plantio de feijão em áreas onde esse herbicida tenha sido aplicado. Além disso, o cultivo dessas espécies após a aplicação desse herbicida contribuirá para a redução do risco de ocorrência de impactos ambientais adversos, como a contaminação de recursos hídricos subterrâneos. No entanto, a continuação desses estudos é necessária, agora, em trabalhos de campo e de laboratório, visando a confirmação dos resultados obtidos.

\section{LITERATURA CITADA}

ACCIOLY, A. M. A.; SIQUEIRA, J. O. Contaminação química e biorremediação do solo. In: NOVAIS, R. F.; ALVAREZ V. V. H.; SCHAEFER, C. E. G. R. Tópicos em ciência do solo. Viçosa: SBCS, 2000. v. 1. p. 299-352.

ANDERSON, T. A.; COATS, J. R. Screening rhizosphere soil samples for the ability to mineralize elevated concentrations of atrazine and metolachlor. J. Environ. Sci. Health, v. B30, p. 473-484, 1995.

ANDERSON, T. A.; KRUGER, E. L.; COATS, J. R. Enhanced degradation of a mixture of three herbicides in the rhizosphere of a herbicide-tolerant plant. Chemosphere, v. 28, p. 1551-1557, 1994.
BOYLE, J. J.; SHANN, J. R. The influence of planting and soil characteristics on mineralization of 2,4,5-T in rhizosphere soil. J. Environ. Qual., v. 27, p. 704-709, 1998.

CUNNINGHAM, S. D.; ANDERSON, T. A.; SCHWAB, A. P. Phytoremediation of soils contaminated with organic pollutants. Adv. Agron., v. 56, p. 55-114, 1996.

EMPRESA BRASILEIRA DE PESQUISA AGROPECUÁRIA - EMBRAPA. Centro Nacional de Pesquisa de Solos. Manual de métodos de análise de solo. 2.ed. Rio de Janeiro: 1997. 212 p.

MACEK, T.; MACKOVÁ, M.; KÁŠ, J. Exploitation of plants for the removal of organics in environmental remediation. Biotechnol. Adv., v. 18, p. 23-34, 2000.

MEYER, R. E.; BOVEY, R. W. Tebuthiuron formulation and placement effects on response of woody plants and soil residue. Weed Sci., v. 36, p. 373-378, 1988.

PIRES, F. R. et al. Fitorremediação de solos contaminados com herbicidas. Planta Daninha, v. 21, n. 2, p. 335-341, 2003.

RODRIGUES, B. N.; ALMEIDA, F. S. Guia de herbicidas. 4.ed. Londrina: Edição dos autores, 1998. $648 \mathrm{p}$.

SCRAMIN, S.; SKORUPA, L. A.; MELO, I. S. Utilização de plantas na remediação de solos contaminados por herbicidas - levantamento da flora existente em áreas de cultivo de cana-de-açúcar. In: MELO, I. S. et al.

Biodegradação. Jaguariúna: EMBRAPA Meio Ambiente, 2001. p. $369-371$.

PERKOVICH, B. S. et al. Enhanced mineralization of $\left[{ }^{14} \mathrm{C}\right]$ atrazine in Kochia scoparia rhizosferic soil from a pesticidecontaminated site. Pestic. Sci., v. 46, p. 391-396, 1996.

RICE, P. J.; ANDERSON, T. A.; COATS, J. R. Phytoremediation of herbicide-contaminated surface water with aquatic plants. In: PHYTOREMEDIATION OF SOIL AND WATER CONTAMINANTS, 1997, Washington. Proceedings... Washington: American Chemical Society, 1997. p. 133-151.

WILSON, P. C.; WHITWELL, T.; KLAINE, S. J. Phytotoxicity, uptake, and distribution of ${ }^{14} \mathrm{C}$-simazine in Canna hybrida 'Yellow King Hunbert'. Environ. Toxicol. Chem., v. 18, p. 1462-1468, 1999.

WILSON, P. C.; WHITWELL, T.; KLAINE, S. J. Phytotoxicity, uptake, and distribution of ${ }^{14} \mathrm{C}$-simazine in Acorus gramenius and Pontederia cordata. Weed Sci., v. 48, p. 701-709, 2000. 